\title{
Microstructure and Magnetic Properties of La-Ca-Sr-Mn-O Perovskite Ceramics
}

\author{
K. Zmorayova*, V. Antal, M. Radusovska, S. Piovarci, J. Kováč, \\ V. KAVECANSKY AND P. DIKO \\ Institute of Experimental Physics SAS, Watsonova 47, 04001 Kosice, Slovak Republic
}

\begin{abstract}
$\mathrm{La}_{0.67} \mathrm{Ca}_{0.33-x} \mathrm{Sr}_{x} \mathrm{MnO}_{3}(x=0.33 ; 0.03 ; 0)(\mathrm{LCSM})$ perovskite magnetocaloric ceramics were prepared by solid state synthesis in air and sintering at $1520^{\circ} \mathrm{C}$. The phase composition was checked by x-ray powder diffraction. Microstructure analyses were done by scanning electron microscopy. The influence of high pressure oxygenation on magnetisation behaviour is shown.
\end{abstract}

DOI: $10.12693 /$ APhysPolA.126.168

PACS: 75.30.Sg, 75.47.Lx, 77.80.Bh, 81.20.Ev, 81.30.Fb

\section{Introduction}

A wide range of different types of materials, from metals to ceramics, exhibit the magnetocaloric effect [1]. Generally, the magnetocaloric effect manifests itself as a reversible increase in temperature when the magnetic material is placed in a magnetic field, and the maximum magnetocaloric effect occurs near the Curie temperature. Magnetic ceramics materials are very stable at room temperature, can be compositionally tuned to adjust the Curie temperature, and do not corrode in water. This makes them an attractive option for use as regenerators for magnetic refrigeration systems. Families of functional materials with a large magnetocaloric effect have been found in several perovskite type manganese oxides such as $\mathrm{Ca}$ - and $\mathrm{Sr}$-doped lanthanum manganites, $\mathrm{La}_{0.67} \mathrm{Ca}_{0.33-x} \mathrm{Sr}_{x} \mathrm{MnO}_{3}(x=0 ; 0.33)$ [2]. These samples show a substantial magnetocaloric effect in a temperature range around their respective Curie temperature, which makes the compounds suitable for air-conditioning and refrigeration applications. By varying the composition parameter $x$ the Curie temperature can be adjusted between $267 \mathrm{~K}(x=0)$ and $369 \mathrm{~K}(x=0.33)$ [2].

In this contribution, the structure, microstructure and the influence of high pressure oxygenation on magnetisation behaviour of $\mathrm{La}_{0.67} \mathrm{Ca}_{0.33-x} \mathrm{Sr}_{x} \mathrm{MnO}_{3}$, prepared by solid state synthesis were investigated.

\section{Experimental}

The $\mathrm{La}_{0.67} \mathrm{Ca}_{0.33-x} \mathrm{Sr}_{x} \mathrm{MnO}_{3} \quad(x=0.33$ (S1); 0.03 (S2); 0 (S3)) perovskite powders were prepared by conventional solid state synthesis in air from the starting materials: lanthanum oxide $\left(\mathrm{La}_{2} \mathrm{O}_{3}\right)$, calcium oxide $(\mathrm{CaO})$, strontium carbonate $\left(\mathrm{SrCO}_{3}\right)$ and manganese oxide $\left(\mathrm{MnO}_{2}\right)$. The powders were mixed in appropriate

*corresponding author; e-mail: zmoray@saske.sk amounts in a mixer for 30 min., and then calcined two times: first at the temperature of $1200^{\circ} \mathrm{C}$ for 16 hours and the second at the temperature of $1300^{\circ} \mathrm{C}$ for 6 hours (heating and cooling rate of $100^{\circ} \mathrm{C} / \mathrm{h}$ in both cases). After each calcination the powders were intensively milled for $15 \mathrm{~min}$. in a friction mill. Then the powders were pressed into cylindrical pellets of $8 \mathrm{~mm}$ in diameter and sintered at $1520{ }^{\circ} \mathrm{C}$ by following temperature programme: heating the sample to sintering temperature with the heating rate $100^{\circ} \mathrm{C} / \mathrm{h}$, dwell at sintering temperature for 2 hours, cooling down to room temperature at $100^{\circ} \mathrm{C} / \mathrm{h}$.

The purity of calcined powders and sintered samples was checked by X-ray diffraction (XRD). The patterns were collected using the $\mathrm{Cu} \mathrm{K}_{\alpha}$ (calcined powders) or Mo $\mathrm{K}_{\alpha}$ (sintered ceramics) wavelength by powder diffractometers. Microstructure analyses were done by scanning electron microscopy (SEM) on fractured surfaces of sintered ceramics.

Small samples, cut for magnetization measurements, were additionally treated by high pressure oxygenation (HPO) at following conditions: $700^{\circ} \mathrm{C}$ for 1 hour, cooling to room temperature at 10 bar pressure of pure oxygen atmosphere.

The thermomagnetic curves were measured by a vibrating-sample magnetometer (VSM) in magnetic field of $200 \mathrm{kA} / \mathrm{m}$ and in temperature range from $140 \mathrm{~K}$ up to $400 \mathrm{~K}$, for the determination of the Curie temperature of samples.

\section{Results and discussion}

The XRD patterns of LCSM powders after calcination and the sintered ceramics are presented in Fig. 1. It can be seen, that not all of the observed peaks can be attributed to the $\mathrm{LaSrMnO}_{3}$ phase after the second calcination. About 16 mass $\%$ belongs to alpha- $\mathrm{La}_{2} \mathrm{O}_{3}(6 \%)$ and $\mathrm{La}\left(\mathrm{MnO}_{3}\right)(10 \%)$ type secondary phases. These secondary phases disappeared after sintering at $1520^{\circ} \mathrm{C}$ and all observed peaks were assigned to the single phase of crystalline perovskite (Fig. 1b). All three studied compositions behaved in the same manner. 

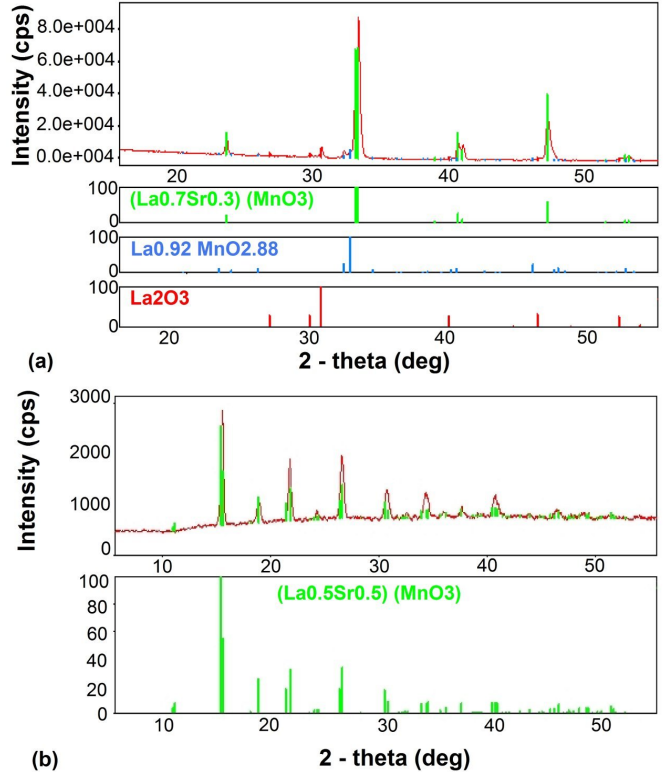

Fig. 1. XRD patterns of $\mathrm{La}_{0.67} \mathrm{Sr}_{0.33} \mathrm{MnO}_{3}$ sample (a) after second calcinations (b) after sintering at 1520 .
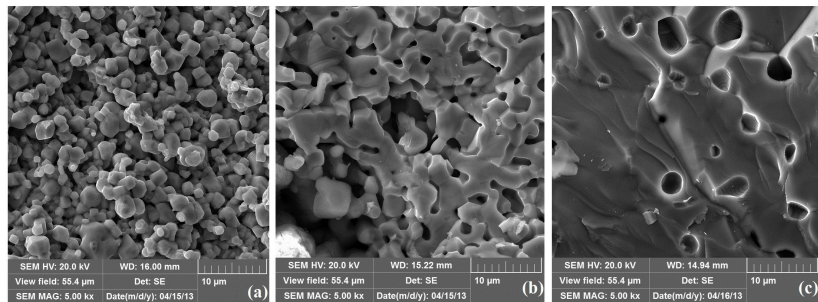

Fig. 2. Fracture surfaces observed by SEM for the samples S1 (a), S2 (b) and S3 (c), sintered at 1520 .

After sintering both, the porosity and the grain size depend on the sample composition, as it is confirmed by observation of fractured surfaces of sintered ceramics by SEM (Fig. 2). From the Fig. 2(c) it can be seen that the sintering and grain growth are the most intensive for the sample S3, where the fracture surface is completely created by transgranular cleavage and exhibits the lowest porosity.

TABLE

$T_{\mathrm{C}}$ of the samples $\mathrm{S} 1, \mathrm{~S} 2, \mathrm{~S} 3$ cooled in air and after HPO.

\begin{tabular}{c|c|c|c}
\hline \hline$T_{\mathrm{C}}(50 \%)$ & $\mathrm{S} 1$ & $\mathrm{~S} 2$ & $\mathrm{~S} 3$ \\
\hline Cooled in air & $356.5 \mathrm{~K}$ & $255.6 \mathrm{~K}$ & $161.9 \mathrm{~K}$ \\
\hline HPO & $353.5 \mathrm{~K}$ & $253.9 \mathrm{~K}$ & $165.1 \mathrm{~K}$
\end{tabular}

The magnetization measurements of Curie temperature, $T_{\mathrm{C}}$, for the samples with different compositions are shown in Fig. 3. We can see that the Curie temperature depends on the nominal composition of $\mathrm{La}_{0.67} \mathrm{Ca}_{0.33-x} \mathrm{Sr}_{x} \mathrm{MnO}_{3}$, as we expected [2], but also on HPO. The HPO leads to lower $T_{\mathrm{C}}$ for the samples $\mathrm{S} 1$ or $\mathrm{S} 2$ and to higher $T_{\mathrm{C}}$ for the sample $\mathrm{S} 3$ (Table).
Such different behaviour can be attributed to changes in oxygen stoichiometry and to consequent changes in the $\mathrm{Mn}^{3+} / \mathrm{Mn}^{4+}$ ratio of $\mathrm{LaSrMnO}_{3}$ phase.
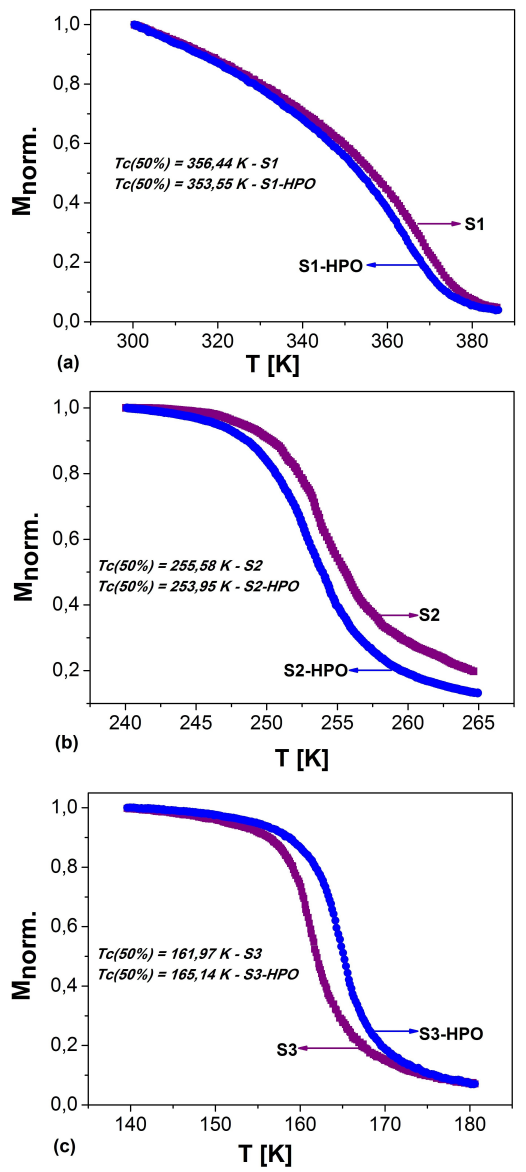

Fig. 3. Curie temperature of the samples (a) S1, (b) S2 and (c) S3 sintered at $1520^{\circ} \mathrm{C}$, cooled in air and after HPO.

\section{Conclusions}

$\mathrm{La}_{0.67} \mathrm{Ca}_{0.33-x} \mathrm{Sr}_{x} \mathrm{MnO}_{3}(x=0,0.03,0.33)$ perovskite samples were prepared by solid state synthesis in air. It is shown that synthesised powders contain some impurity phases, which further react at sintering of ceramic bulk samples at higher temperatures. The magnetisation measurements confirmed expected behaviour of Curie temperature with Sr substitution and have shown the influence of high pressure oxygenation.

\section{Acknowledgments}

This work was realized within the framework of the projects: ITMS 26220120019, ITMS 26220120035, ITMS 26220220061, ITMS 26220220041, VEGA project No. 2/0090/13 and 1/0861/12, SAS Centre of Excellence: CFNT MVEP.

\section{References}

[1] K.A. Gschneidner, V.K. Pecharsky, A.O. Tsokol, Rep. Prog. Phys. 68, 1479 (2005).

[2] A.R. Dinesen, S. Linderoth and S. Mørup, J. Phys.: Condens. Matter 17, 6257 (2005). 